\title{
Holographic progressive lenses
}

Jannik Trapp, Manuel Decker, Jörg Petschulat, Thomas Pertsch, Toufic Jabbour

Jannik Trapp, Manuel Decker, Jörg Petschulat, Thomas Pertsch, Toufic G. Jabbour, "Holographic progressive lenses," Proc. SPIE 10745, Current Developments in Lens Design and Optical Engineering XIX, 107450G (17 September 2018); doi: 10.1117/12.2503173

Event: SPIE Optical Engineering + Applications, 2018, San Diego, California, United States 


\title{
Holographic progressive lenses
}

\author{
Jannik M. Trapp*a,b, Manuel Decker ${ }^{\mathrm{a}}$, Jörg Petschulat ${ }^{\mathrm{c}}$, Thomas Pertsch ${ }^{\mathrm{b}, \mathrm{d}}$, Toufic G. Jabbour ${ }^{\mathrm{e}}$, \\ aCorporate Research \& Technology, Carl Zeiss AG, Carl Zeiss Promenade 10, 07745 Jena, Germany \\ ${ }^{b}$ Institute of Applied Physics, Abbe Center of Photonics, Friedrich Schiller University Jena, Albert- \\ Einstein-Str. 15, 07745 Jena, Germany \\ ${ }^{\mathrm{c}}$ Global Research and Technology, Semiconductor Mask Solutions, Carl Zeiss SMT GmbH, Carl \\ Zeiss Promenade 10, 07745 Jena, Germany \\ ${ }^{\mathrm{d}}$ Fraunhofer Institute for Applied Optics and Precision Engineering, Albert-Einstein-Str. 7, 07745 \\ Jena, Germany \\ ${ }^{\mathrm{e}}$ Corporate Research \& Technology, Carl Zeiss AG, Carl-Zeiss-Straße 22, 73447 Oberkochen, \\ Germany
}

\begin{abstract}
Progressive addition lenses (PALs) are realized as optical freeform surfaces by complex manufacturing and inspection processes. In turn, holographic optical elements (HOEs) enable fast and easy fabrication in other applications. Here we present a design method for the combination of both; HOEs that are designed to fulfill the optical function of PALs. We describe how inherent limitations of HOEs, such as angular and wavelength selectivity as well as grating dispersion can be overcome. We show that holographic PALs can be optimized to have a distribution of spherical power and astigmatism, which is a qualitative replication of the performance of their refractive counterparts. The design rules we identify are shown for PALs but have the potential to improve other applications of HOEs as well.
\end{abstract}

Keywords: Holography, Progressive Lenses, Eyeglasses, Diffractive Optics, Gratings

*jannik.trapp@zeiss.com; zeiss.com

\section{INTRODUCTION}

A significant share of the human population suffers from an age-related eye condition known as presbyopia. It is a stiffening process of the crystalline eye lens, which leads to a decreased ability of the eye lens to change its curvature (accommodation) as presbyopia progresses with age [1]. This makes focusing for near vision increasingly difficult. As a result, the eye requires additional spherical power for near vision tasks. For emmetropic eyes, presbyopia can be compensated for by reading glasses. They supply the user with the required additional spherical power for near vision tasks, such as reading or computer work, but have the drawback that the lenses need to be changed between tasks.

More convenient and for ametropic eyes presbyopia usually is treated with progressive addition lenses (PALs). At this point, the user requires different additional spherical powers for varying imaging distances. Refractive PALs meet the need of spherical power variation by providing zones for near and far vision with different lens curvatures, i.e., different spherical powers. Figure 1(a) shows the distribution of spherical power over a lens surface of a sample PAL. This PAL has a diameter of $50 \mathrm{~mm}$ and is designed for a late-stage presbyopic but emmetropic eye. The upper part of the lens around $5<y<25 \mathrm{~mm}$ is designed for far vision, which does not require additional spherical power. Therefore, this region has a spherical power of zero diopters (dpt.). For users with additional refractive errors the corresponding PAL design needs to include the target prescription in addition to the distribution of additional spherical power. The lower part of the lens, shown as the yellow area at $-8<x<8 \mathrm{~mm}$ and $-25<y<-10 \mathrm{~mm}$, is designed for near vision and has the highest amount of spherical power of the lens, here chosen as $2.5 \mathrm{dpt}$. The zones for near and far vision are connected by a continuous increase of spherical power known as the progressive corridor, which is located at $-3<x<3 \mathrm{~mm}$ and $-10<y<5 \mathrm{~mm}$. As known from Minkwitz's theorem [2], increasing spherical power along one direction at a certain rate causes astigmatism increasing at twice the rate in the perpendicular direction. The consequence for PALs is that the in-plane regions orthogonal to the progressive corridor distort vision due to high astigmatism [3,4]. Therefore, it is a requirement for PALs that the astigmatism in the viewing zones and within the progressive corridor is minimal. Figure 1(b) shows the distribution of

Current Developments in Lens Design and Optical Engineering XIX, edited by R. Barry Johnson, Virendra N. Mahajan,

Proc. of SPIE Vol. 10745, 107450G @ The Authors. Published under a Creative Commons Attribution

CC BY 3.0 License doi: 10.1117/12.2503173

Proc. of SPIE Vol. 10745 107450G-1 
astigmatism over the lens surface for the PAL whose spherical power is shown in part (a). Here, astigmatism is expressed as the focus difference between two orthogonal axes in dpt. Clearly, astigmatism is low for the two viewing zones and within the progressive corridor, which are all blue areas. The respective distributions of spherical power and astigmatism of the PAL shown in figure 1 will serve as a benchmark for the holographic PAL (hPAL) design.

a)

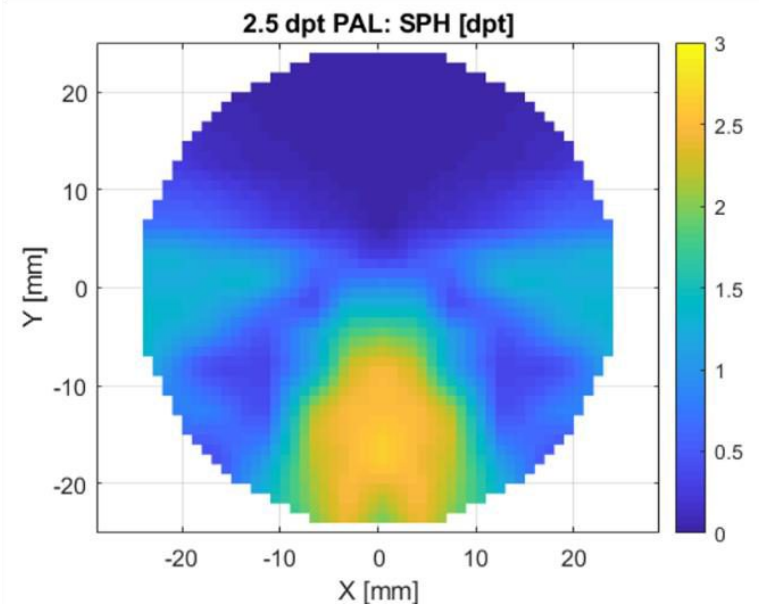

b)

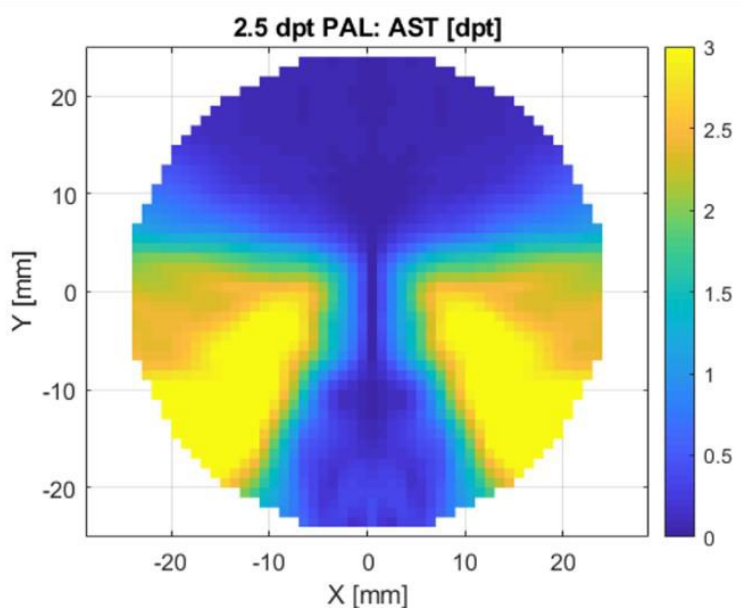

Figure 1: Spherical power (a) and astigmatism (b) of a PAL designed for a late-stage presbyopic but emmetropic eye with a lens diameter of $50 \mathrm{~mm}$ and spherical powers of up to $2.5 \mathrm{dpt}$. The spherical power distribution consists of a zone for far vision at a spherical power of about $0 \mathrm{dpt}$. in the upper half of the lens and a zone for near vision at about $2.5 \mathrm{dpt}$. in the lower part of the lens. The two zones are connected by a smooth increase in spherical power known as the progressive corridor.

PAL manufacturing requires high precision manufacturing processes to achieve the continuous gradient in surface curvature responsible for the spatially varying power. This usually is obtained by computer-numerically controlled (CNC) machines with complex soft polishing opposed to the easier hard polishing of spherical lenses [5]. Therefore, it is desirable to provide a solution where a custom PAL functionality can be imprinted on a standard single-vision lens substrate.

This is a possible application for holographic optical elements (HOEs) since their optical function can be realized in a thin film and can be tuned to work with curvature [6-8]. Furthermore, state of the art holographic materials and processing techniques enable fast and scalable mass production [9-11]. In this work, we consider materials available as thin foils with a thin layer of instant developing photopolymer, which does not require wet chemical processes.

There are four basic challenges for using HOEs in eyeglasses:

1. Dispersion compensation: HOEs are gratings and therefore induce grating dispersion. To minimize angular color error $(\mathrm{CE})$, this grating dispersion needs to be controlled.

2. Wavelength bandwidth: Achieving high diffraction efficiency (DE) over the entire visible spectrum of light (VIS) of the human eye [12] is necessary for color vision.

3. Angular bandwidth: Achieving high DE over a certain angular bandwidth is necessary to account for the rolling eye.

4. Optical function: Recreating the spherical power distribution of a PAL with minimal astigmatism is necessary to solve fulfill the purpose of an hPAL.

Challenges 2) and 3) are very critical, as the wavelength and angular bandwidth of HOEs is typically very limited [13-15]. Furthermore, wavelength and angular bandwidth depend on the same grating parameters. Therefore, it needs to be ensured that challenges 2) and 3) are accomplished at the same time.

In this contribution, we present an optimization method for hPALs. We address all mentioned challenges and provide a performance evaluation of a sample hPAL design in terms of spherical power and astigmatism distribution. Finally, we benchmark the hPAL with its refractive counterpart introduced in figure 1.

\section{METHODS}

In this section, we present our design methods to address the four basic challenges for the use of HOEs as hPALs. 
We choose an optical system made up of two HOEs and the rolling eye of the observer as illustrated in Figure 2(a) as the starting point for our considerations. Here, we only consider a 2D case without loss of generality. In the calculations presented later, the HOEs are embedded into a polymer substrate, which is not shown here for simplicity. When using eyeglasses, the eye of the observer rotates as indicated by the rotation angle $\theta$. This creates a continuum of viewing directions. We consider the ray connecting the rotational center of the eye $\mathrm{M}$ with the center of the pupil to be the principal ray of the respective viewing direction. Each principal ray can be treated as the optical axis of the corresponding viewing direction. Here, the principal rays for the eye looking straight ahead (rotation angle zero) and looking downwards at the indicated angle $\theta$ are indicated by arrows. The eye pupil acts as the aperture of the optical system and moves as the eye rotates. This movement of the optical axis and system aperture is a significant difference between eyeglasses and other optical systems and leads to different requirements for the optical design approach. In our case, this means that the HOE design has to be optimized for each viewing direction.

\subsection{Grating dispersion}

Our approach to minimize grating dispersion is to use a tandem of two HOEs as shown in figure 2(a). We choose this configuration because grating dispersion induced by a first grating can be compensated by the dispersion induced by a second grating operating in the opposite diffraction order with a similar grating period [16]. The Ewald spheres shown in figure 2(b) visualize the HOEs' grating vectors (red) for the HOEs inducing the direction changes experienced by the lower principal ray from figure 2(a). The grating vectors $\left|\overrightarrow{K_{B}}\right|$ and $\left|\overrightarrow{K_{A}}\right|$ belonging to HOE B and HOE A, respectively, are of equal magnitude and opposite direction. This visualizes that the HOEs do not induce ray direction changes in the default configuration. This is important, as the user of an eyeglass wants to look straight ahead with limited prismatic effect. An additional advantage of using this tandem setup is that it allows decoupling the deflection angle from the grating vector. At a later stage, we will use this in our considerations for the HOEs' DEs.

a)

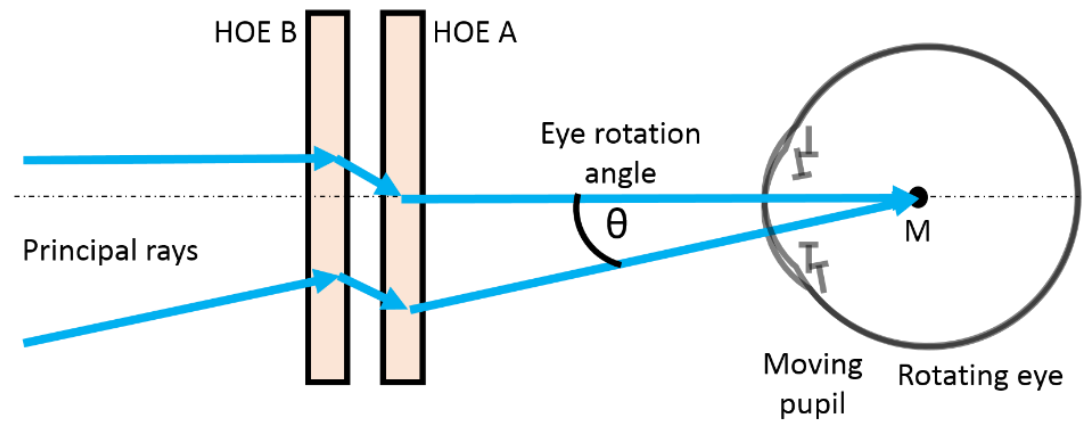

b)
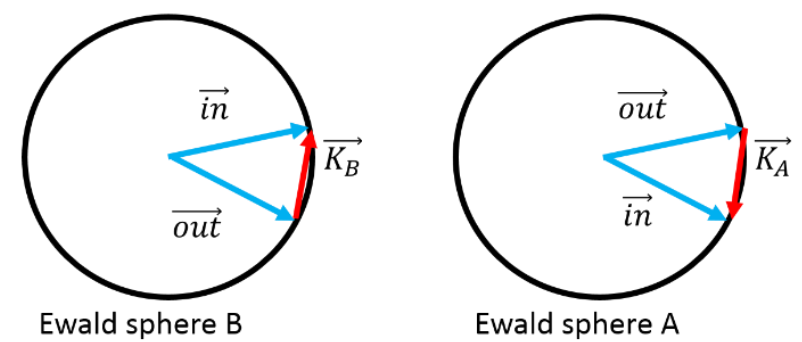

Figure 2: (a) Illustration of the optical system consisting of two HOEs and the rotating eye of the observer. The difference in rotation angle $\theta$ is shown for two sample viewing directions. A principal ray connecting the rotational center of the eye $\mathrm{M}$ and the center of the pupil is drawn for both sample viewing directions. (b) Ewald spheres showing the grating vectors (red) for HOE A and B at the positions of the ray path of the lower principal ray. As the two grating vectors are of equal magnitude and opposite direction, the grating dispersion induced by one HOE is compensated by the grating dispersion induced by the other HOE.

\subsection{HOE bandwidth}

As a first step, we estimate the required angular bandwidth for our HOE. For this purpose, we consider a parallel bundle of rays entering the eyes pupil at an arbitrary viewing direction with the principal ray of the viewing direction being the center ray in the bundle. Later in this section, we will ensure that the available angular bandwidth at each position on the lens is centered on the corresponding principal ray. Since the DE at each lens position is optimized for the corresponding 
principal ray, DE is maximal for the center ray in the bundle. Following the same logic, the outer rays go through lens positions, whose DE is optimized for other principal rays. The direction difference between the outer rays in the bundle and these principal rays describes the relevant angular detuning. The angular detuning increases towards the ends of the ray bundle and is limited by the pupil, which constrains the size of the ray bundle. For a pupil diameter of $2 \mathrm{~mm}$, which is reasonable for day light vision [12], and typical geometries for the HOE and the eye, we find that an angular bandwidth of about $3.4^{\circ}\left(+/-1.7^{\circ}\right)$ around each principal ray is required.

We investigate DE as a function of HOE grating period, thickness and index modulation using the Fourier Modal Method (FMM) [17] to find a HOE configuration with the required angular and wavelength bandwidth at DE values above 0.7. Our first step is to optimize DE as a function of wavelength. We find that at a thickness of $25 \mu \mathrm{m}$ and a refractive index modulation of $0.02^{1}$, which is easily achievable with state-of-the-art materials $[19,20]$, combined with grating periods between 2.0 and $2.7 \mu \mathrm{m}$ we achieve the required wavelength bandwidth. We found the preferred grating period value for highest DE to be $2.4 \mu \mathrm{m}$. While this approach allowed us to achieve the desired wavelength bandwidth, the corresponding angular bandwidth is far below the requirement. We address this issues by a procedure we refer to as multiplexing, which is a known way to increase the bandwidth of HOEs [21]. Here, we decide to multiplex two HOEs into the same holographic film, what leads to a significant increase in angular bandwidth. With this approach, we are able to achieve the required angular and wavelength bandwidth at the same time.

To provide maximum DE for all viewing directions, we have to ensure that the available angular bandwidth at all lens positions is centered on the corresponding principal ray. The grating vector of HOEs is defined as $\vec{K}=\frac{2 \pi}{\Lambda}\left(\begin{array}{l}\cos \varphi \\ \sin \varphi\end{array}\right)$ with the grating period $\Lambda$, slant angle $\varphi$, the $x$ coordinate denoting the direction of propagation and the $y$ coordinate being orthogonal to the plane of incidence [13] (assuming invariance in the $z$ coordinate). Manipulating the slant angle $\varphi$ allows to shift DE as a function of angle. We use this to ensure that the angular bandwidth is centered on the principal ray for each viewing direction. It should be noted that this procedure only shifts the angular bandwidth, the magnitude of angular and wavelength bandwidth are not affected.

\subsection{Recreating the optical function of a PAL}

The spherical power of HOEs depends on locally varying grating periods and slant angles. In order to design hPALs with a custom spherical power profile, we want to find a HOE parametrization to locally control grating periods and slant angle over the lens surface. For this purpose, we implement the HOEs as surface gratings. This can be done for the purposes of ray tracing as the deflection angle of volume gratings such as HOEs is the same as in surface gratings. However, this means that the implemented grating period is actually the one on the grating surface, not the grating period in the volume. The surface grating period can be obtained by projecting the grating vector onto the grating surface as $\overrightarrow{K_{y}}=\frac{2 \pi}{\Lambda}\left(\begin{array}{c}0 \\ \sin \varphi\end{array}\right)$. Again, we consider a $2 \mathrm{D}$ case without loss of generality. The surface grating period obtained this way is $\Lambda_{y}=\Lambda / \sin \varphi$. In our implementation, we always choose the slant angle $\varphi$ so that the available bandwidth is centered on the principal rays as discussed earlier. The surface gratings representing the HOEs are then parametrized by global polynomials, which describe variations of the grating period and slant angle over the lens surface. The coefficients of the polynomial can then be varied to achieve different distributions of spherical power over the lens surface.

\subsection{Optical design}

We implement our design method described above in an optical design software. We set up an optical system consisting of an HOE tandem and the rotating eye of the observer. The HOEs are sandwiched into a $1.4 \mathrm{~mm}$ polymer substrate with a refractive index of 1.59. For each considered viewing direction, we place a point source at an appropriate distance for either near, intermediate or far vision in object space. We implement the HOEs as parametrized surface gratings. We set target values for the distribution of spherical power and astigmatism based on the benchmark device shown in figure 1 . We then use a damped least-squares algorithm to optimize the HOEs for all viewing directions simultaneously.

\footnotetext{
${ }^{1}$ HOEs are gratings characterized by modulation of the refractive index in a volume material [18] Kogelnik, H.,

"Coupled wave theory for thick hologram gratings," The Bell System Technical Journal 48, 2909-2947 (1969).
} 


\section{RESULTS AND DISCUSSION}

To evaluate whether our hPAL design qualitatively replicates the standard PAL, we compare their spherical power and astigmatism. The distributions of spherical power and astigmatism are shown in figure 3(a) and (b), respectively, while those corresponding to the standard PAL are shown in figure 1. For better visualization of the difference between the two designs, the difference between the spherical power and astigmatism of the PAL and the hPAL are shown in figure 3(c) and $(\mathrm{d})$, respectively.

We find that the distributions of spherical power of the two devices are very similar. The zone for near vision of the hPAL located at $-22<y<-11 \mathrm{~mm}$ and $-5<x<5 \mathrm{~mm}$ is a bit smaller than the corresponding zone in the benchmark device located at $-20<y<-8 \mathrm{~mm}$ and $-7<x<7 \mathrm{~mm}$. The shape of the zone for far vision, which is found at about $y>5 \mathrm{~mm}$ in both devices, appears slightly reduced in size in the hPAL as well. However, the basic functionality of the PAL to provide zones for near and far vision with a continuous increase of spherical power connecting the two is recreated by the hPAL. This is verified by figure 3(c), which illustrates that larger differences between the spherical power distributions of the PAL and the hPAL only occur outside of the zones for near and far vision and the progressive corridor.

The distributions of astigmatism of the two devices are quite different at first glance. Clearly, the overall amount of astigmatism in the PAL is much higher than in the hPAL. Upon closer inspection, we find that the astigmatism in the progressive corridor and the zones for near and far distance vision in the hPAL is very comparable to the astigmatism in the corresponding zones of the PAL. The main difference between the two devices is that to the left and right of the near vision zone, the hPAL has a very smooth increase of astigmatism, while the corresponding zone of the PAL is limited by rapidly increasing astigmatism. This assessment can be verified by the difference plot shown in figure 3(d), where the larger differences between the astigmatism of the PAL and the hPAL are located outside of the zones for near and far vision and the progressive corridor.

In summary, we find that our hPAL design qualitatively replicates the distributions of spherical power and astigmatism of the standard PAL. 
a)

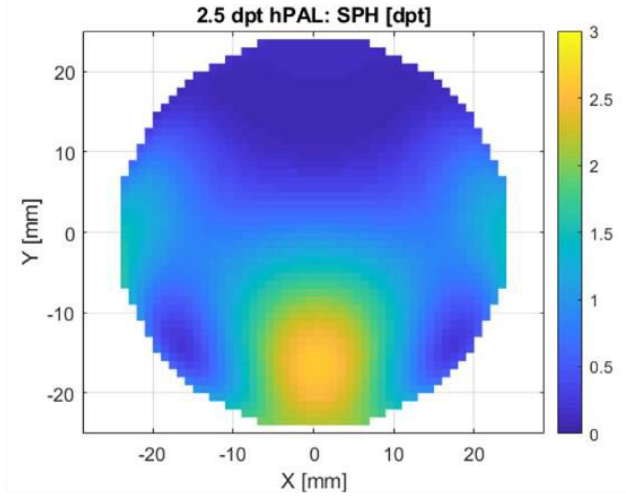

c)

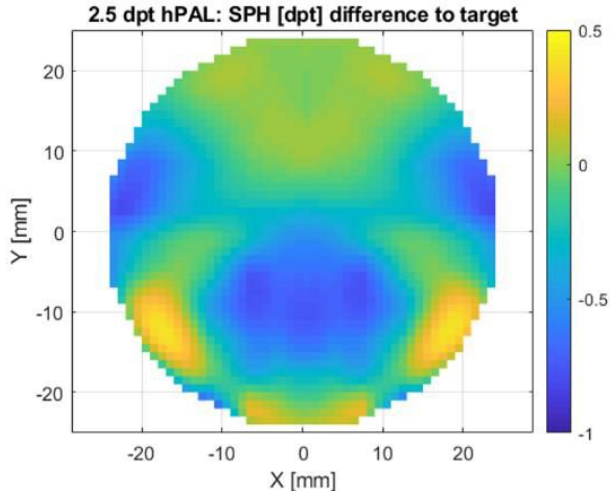

b)

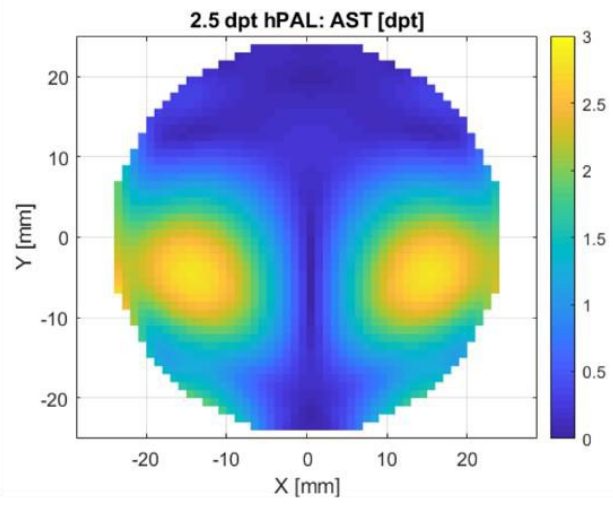

d)

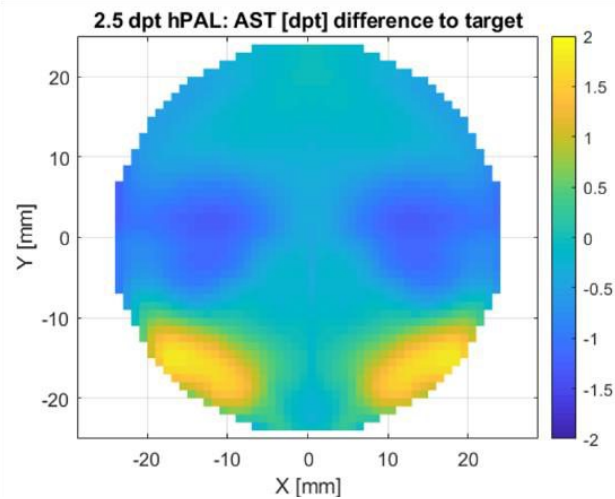

Figure 3: Spherical power (a) and astigmatism (b) of an hPAL with additional spherical power up to $2.5 \mathrm{dpt}$. The difference of spherical power (c) and astigmatism (d) between the PAL shown in figure 1 and the hPAL illustrate that the near and far vision zones and the progressive corridor are very similar and that strong differences between the two devices only occur outside of these zones.

We evaluate the CE of our hPAL design. We benchmark the CE of the hPAL by comparing it to the CE of a unifocal -4 dpt. eyeglass, whose imaging performance exhibits $\mathrm{CE}$ induced color fringes of more than twice the perception threshold for color fringes [22]. We find that the CE of the hPAL presented here currently exceeds the one of the benchmark device.

\section{CONCLUSION}

We have presented a design method how HOEs can be utilized as PALs. The found rules have been applied to design a HOE-based PAL, which qualitatively replicates the spherical power and astigmatism distribution of standard PAL. As we work with HOEs made from flat foils, our HOE designs are suitable candidates for fast and scalable manufacturing processes. Therefore, our work indicates that HOEs can be a viable alternative to standard eyeglasses in some specialized applications such as PALs. The design method presented here has the potential to improve other applications of HOEs as well.

\section{FUNDING}

This project has received funding from the EU's Horizon 2020 research and innovation programme under the Marie Skłodowska-Curie grant agreement No 675745.

\section{ACKNOWLEDGEMENTS}

We thank Philipp Jester, Helmut Wietschorke, Gerhard Kelch and Katharina Rifai from Carl Zeiss Vision GmbH for helpful discussions and providing data on standard progressive addition lenses. 
We thank Christoph Menke from Carl Zeiss AG for helpful discussions on optical design and optimization.

\section{REFERENCES}

[1] Glasser, A., and Campbell, M. C. W., "Biometric, optical and physical changes in the isolated human crystalline lens with age in relation to presbyopia," Vision Research 39, 1991-2015 (1999).

[2] Minkwitz, G., "Über den Flächenastigmatismus Bei Gewissen Symmetrischen Asphären," Optica Acta: International Journal of Optics 10, 223-227 (1963).

[3] Meister, D. J., and Fisher, S. W., "Progress in the spectacle correction of presbyopia. Part 1: Design and development of progressive lenses," Clinical and Experimental Optometry 91, 240-250 (2008).

[4] Sheedy, J. E., Campbell, C., King-Smith, E., and Hayes, J. R., "Progressive Powered Lenses: the Minkwitz Theorem," Optometry and Vision Science 82, 916-922 (2005).

[5] Meister, D. J., and Fisher, S. W., "Progress in the spectacle correction of presbyopia. Part 2: Modern progressive lens technologies," Clinical and Experimental Optometry 91, 251-264 (2008).

[6] Osten, W., Faridian, A., Gao, P., Körner, K., Naik, D., Pedrini, G., Singh, A. K., Takeda, M., and Wilke, M., "Recent advances in digital holography [Invited]," Appl. Opt. 53, G44-G63 (2014).

[7] Rostykus, M., Rossi, M., and Moser, C., "Compact lensless subpixel resolution large field of view microscope," Opt. Lett. 43, 1654-1657 (2018).

[8] Li, G., Lee, D., Jeong, Y., Cho, J., and Lee, B., "Holographic display for see-through augmented reality using mirror-lens holographic optical element," Opt. Lett. 41, 2486-2489 (2016).

[9] Jurbergs, D., Bruder, F.-K., Deuber, F., Fäcke, T., Hagen, R., Hönel, D., Rölle, T., Weiser, M.-S., and Volkov, A., "New recording materials for the holographic industry," (2009), pp. 72330K-72330K-72310. [10] Russo, J. M., Dimov, F., Padiyar, J., and Coe-Sullivan, S., "Mass production of holographic transparent components for augmented and virtual reality applications," in SPIE Digital Optical Technologies(SPIE2017), p. 9.

[11] Bruder, F.-K., Fäcke, T., Grote, F., Hagen, R., Hönel, D., Koch, E., Rewitz, C., Walze, G., and Wewer, B., "Mass production of volume holographic optical elements (vHOEs) using Bayfol(R) HX photopolymer film in a roll-to-roll copy process," in SPIE OPTO(SPIE2017), p. 20.

[12] Gross, H., Blechinger, F., and Achtner, B., [Handbook of Optical Systems, Survey of Optical Instruments], Wiley, (2008).

[13] David, B.-R., "Understanding Diffraction in Volume Gratings and Holograms," in Holography - Basic Principles and Contemporary Applications, E. Mihaylova, ed. (InTech, 2013), p. Ch. 01.

[14] Marín-Sáez, J., Atencia, J., Chemisana, D., and Collados, M.-V., "Characterization of volume holographic optical elements recorded in Bayfol HX photopolymer for solar photovoltaic applications," Opt. Express 24, A720-A730 (2016).

[15] Han, J., Liu, J., Yao, X., and Wang, Y., "Portable waveguide display system with a large field of view by integrating freeform elements and volume holograms," Opt. Express 23, 3534-3549 (2015).

[16] Palmer, C. A., [Diffraction Grating Handbook], Richardson Grating Laboratory, (2000).

[17] Li, L., "New formulation of the Fourier modal method for crossed surface-relief gratings," J. Opt. Soc. Am. A $14,2758-2767$ (1997).

[18] Kogelnik, H., "Coupled wave theory for thick hologram gratings," The Bell System Technical Journal 48, 2909-2947 (1969).

[19] Zanutta, A., Orselli, E., Fäcke, T., and Bianco, A., "Photopolymer materials for volume phase holographic optical elements," in International Conference on Space Optics - ICSO 2016(SPIE2017), p. 9.

[20] Chang, B. J., "Dichromated Gelatin Holograms And Their Applications," (SPIE1980), p. 7.

[21] S. Altmeyer, Y. H., P. Thiée, J. Matrisch, M. Wallentin and J. Silbermann, "Multiplexing of transmission holograms in photopolymer," in DGaO Proceedings(2013).

[22] Goersch, H., [Wörterbuch der Optometrie], DOZ-Verlag, Heidelberg (2004). 